\title{
Safety Lines Detection as a Means of NAVIGATION IN INDUSTRIAL FACILITIES
}

\author{
Karamanos Xenofon, Bechtsis Dimitrios, \\ Vlachos Dimitrios \& Mastos Theofilos
}
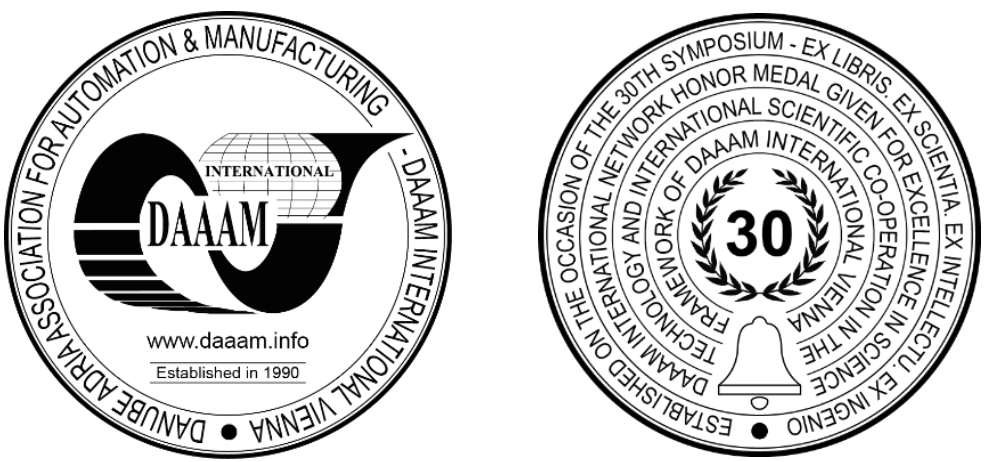

This Publication has to be referred as: Karamanos, X[enofon]; Bechtsis, D[imitrios]; Vlachos, D[imitrios] \& Mastos, T[heofilos] (2021). Safety Lines Detection as a Means of Navigation in Industrial Facilities, Proceedings of the 32nd DAAAM International Symposium, pp.0256-0261, B. Katalinic (Ed.), Published by DAAAM International, ISBN 9783-902734-33-4, ISSN 1726-9679, Vienna, Austria

DOI: $10.2507 / 32$ nd.daaam.proceedings.036

\begin{abstract}
Autonomous Mobile Robots (AMRs) are becoming a crucial part of industrial environments. Path planning and navigation activities are still core components of AMR literature. AGV navigation methods traditionally utilize floor markings, while state of the art systems are using cameras to identify the vehicle's position and direction and map the facility layout. This paper focuses on how an AMR with an RGB camera could take advantage of floor markings and improve the vehicle's navigation and safety conditions, especially in manufacturing environments with large empty spaces where cameras have limited positioning capabilities. The system is built on top of the Robot Operating System (ROS). A safety line detector is trained to recognise floor markings and the AMR uses them as a guidance line in order to safely navigate at a specific distance from it. The detector is a MASK-RCNN network, trained on real-world images captured at the industrial facility. Each image was manually annotated in order to feed the network. The proposed system was extensively tested in simulation and in real world environment.
\end{abstract}

Keywords: ROS; MASK-RCNN; Navigation; Intralogistics; Lane Detection

\section{Introduction}

Autonomous Mobile Robots (AMRs) are becoming increasingly valuable assets to warehouses and industrial facilities, as part of the fourth industrial revolution (Industry 4.0). AMRs can be used for intra-logistics and material handling activities without being manipulated by human agents. Incorporating AMRs can increase productivity and flexibility [1], reduce costs and enable agile manufacturing. Moreover AMRs flexibility allows them to be easily customized for many tasks. In this context, AMRs must be able to work along with human agents and safely travel through complex layouts and industrial spaces. Of course, simulations tools verify the added value and help identify the operational needs of facilities[2] . Additionally, visual tools are proposed in order to facilitate the AMRs by non-expert personnel [3].

Most industrial facilities are required to clearly indicate goods, the transfer zones that are used as buffers and the cells were the workstations are installed. Colour labelling is one of the most efficient ways to divide the different segments of a workplace and is also considered as a part of 5S [4] and lean manufacturing. 
Colour coding gives some additional benefits such as speeding up visual search, emphasizing information like aisleway and exit paths and notates hazardous areas. A main aspect of this colour labelling is to separate forklifts' traffic flows from pedestrians' flows. Pedestrians' pathways are clearly marked and visible as the forklifts' pathways. This ensures a significant safety level and reduces the accidents by making the workspace safer, more pleasant, and well-organized [5]. The proposed research work, uses the already established floor markings in order to facilitate the vehicle's navigation with increased safety. Recognizing the floor markings and safety lines, improves safety and navigation of the AMR, and this can be achieved with a single RGB camera. The rest of this article is organized as follows. In Section 2, a brief literature review on AMR systems and lane detection systems is presented, Section 3 analyses the proposed system and Section 4 focuses on the experimental results. Finally, Section 5 presents the conclusions and future research directions.

\section{Literature Review}

Many methods, are actually used to detect a lane or a lane's lines in a road or an industrial transportation path. There are many image processing algorithms that can be used to detect lane lines such as feature-based detections, model-based and more modern ones with semantic segmentation networks.

A feature-based method operates most of the time using the characteristics of line shape and/or colour features. A simple algorithm is thresholding [6], global or dynamic. In global thresholding algorithms, grey level histogram of the image is used and the algorithm includes this information to detect lane markers. This is not very effective, though, due to that a similar grey scale histogram is required [7]. Other methods, include a pre-process step, like edge detectors, such as Canny, Gabor, Sobel and Laplacian operators. After the edge detection is completed, image enhancement is performed to improve the next step of lane line detection. The algorithms are then able to detect lines based on the vanishing point estimation [[8] and curve fitting [9].

Model-based methods are usually trying to fit the detection into some geometric or curve model. [10] utilizes the camera parameters to form a lane geometrical model. The initial stage is to estimate the vanishing point, followed by the lane boundary detection using Canny edge detection and Hough transforms. The final step is to match the lane's curvature from a set of candidates. In [11] the authors propose a lane detection based on spatiotemporal images. Points of lanes are detected by aligning scanlines along the time axis, followed by a Hough transform and the prediction of an offset for the scanline in the next frame. All the points detected are fitted to a cubic curve model.

Deep neural networks are not a recent breakthrough [12]although computational advances enabled the heavy usage of such networks. The semantic segmentation network is based on neural deep convolutional networks. Its main purpose is to segment the pixels in an image into distinguishable targets, like roads, persons, pavements and others. The authors in [13] based their method on Convolution Neural Networks (CNN). The lane line segmentation is split into two stages. The first stage is responsible to semantically segment the lane boundaries and the second to classify the lane. [14]performs lane detection using a modified encoder-decoder based model ENet [15] with two distinct branches for binary and instance segmentation. Binary segmentation is performed to detect all lines in the frame. Instance segmentation outputs an embedding for each lane pixel so that the distance between pixel embedding belonging to the same lane is small, where to different lanes is maximized.

\section{Safety Line Detection System}

Safety Line Detection System is a pretrained Mask-RCNN [16] model, trained further on images taken from industrial warehouses. First, we must collect images (frames) to train the system. Then, the network is trained, and finally its performance is validated in order to be deployed on the robot and perform in real-world environments. The whole pipeline of training the system is explained below.

\subsection{Annotating Data}

To train the system, we manually annotated and labelled 1145 frames. Due to that Mask-RCNN is already pretrained, there is no need for huge amount of data. The whole annotation process was conducted with a user-friendly open-source tool called VGG Image Annotator [17] . VGG is a web-based tool and offers various shapes to label specific regions within the images, namely rectangles, circles, polygons and offers multiple types of attributes (indicatively text, checkboxes, radio buttons).

After the annotation is completed as indicated in Figure 1, the tool allows us to save the project for future use and export annotations to various formats like CSV, JSON and COCO [18] annotation format. We export the annotations as $\mathrm{COCO}$, since our next step, training, requires the COCO format. COCO format is a type of JSON file type with specific fields for different annotation types. 


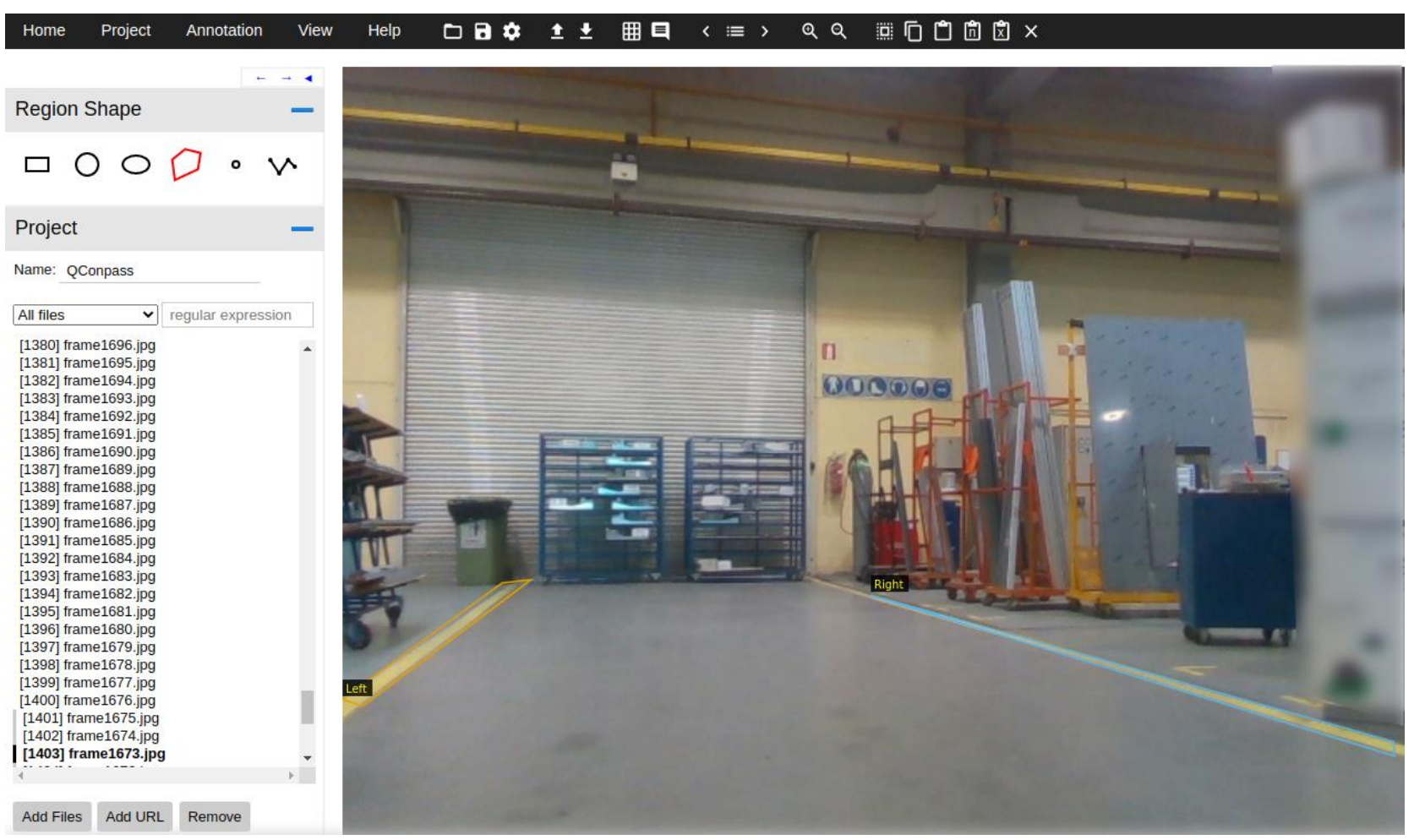

Fig. 1. VGG Image annotator GUI with two safety lines annotations (left and right lines)

\subsection{Training MASK-RCNN}

Having the annotations, the next step of the pipeline is to actually train it. This is possible with frameworks such as TensorFlow [19] and PyTorch [20]. The PyTorch framework was selected due to its clarity and being developer friendly. Also, it is written with keeping in mind the pythonic programming style native python and as a result, it is an objectoriented framework.

At the beginning, the annotation that was created in the previous step must be used to create the Dataset that the network will be trained on. PyTorch offers the ability to create custom datasets in an extremely easy and friendly way. It provides various classes that a user can derive from conforming to different annotation formats. Since the annotations was exported in $\mathrm{COCO}$ format, we can create a new class deriving from the torchvision.datasets. CocoDetection class. We then load our annotations with the package pycocotools. We also split the dataset to a training and a validation dataset to prevent overfitting the model.

The next step is to define our model, specifically Mask-RCNN model. Mask-RCNN is an extension to Faster-RCNN [21]. Both can use as backbone, different feature detectors. such as Resnet $\{50,101\}$ [22], Yolov3 [23] and others. FasterRCNN can detect regions of interest and place bounding boxes around them. Mask-RCNN adds an extra branch that can not only place that bounding box but also segment the instance and provide a mask of it. Moreover, all this is possible only with a small overhead to Faster-RCNN. Again, the simplicity and module reusability of PyTorch, allows the user to create the model very easily and modify specific parameters for any use case, such as number of classes to detect and number of hidden layers. We then define the optimizer and learning rate scheduler, where Stochastic Gradient Descent (SGD) and Step Learning Rate (StepLR) classes were used respectively.

Training the model was performed on a CUDA capable device, a GeForce GTX 1060 6GB/PCIe/SSE2 and total time for an epoch was around 2 min and 10 secs with batch size of 4 . The system was trained for a total of 8 epoch with the final metrics of average precision shown in Table 1.

\begin{tabular}{|c|c|c|c|c|}
\hline Safety Line Detection & Backbone & AP & AP50 & AP $_{\mathbf{7 5}}$ \\
\hline Bounding Box & Resnet50-FPN & 0.834 & 0.987 & 0.935 \\
\hline Segmentation & Resnet50-FPN & 0.451 & 0.872 & 0.448 \\
\hline
\end{tabular}

Table 1. Average Precision (AP) Metrics 


\subsection{Deploy on Robot}

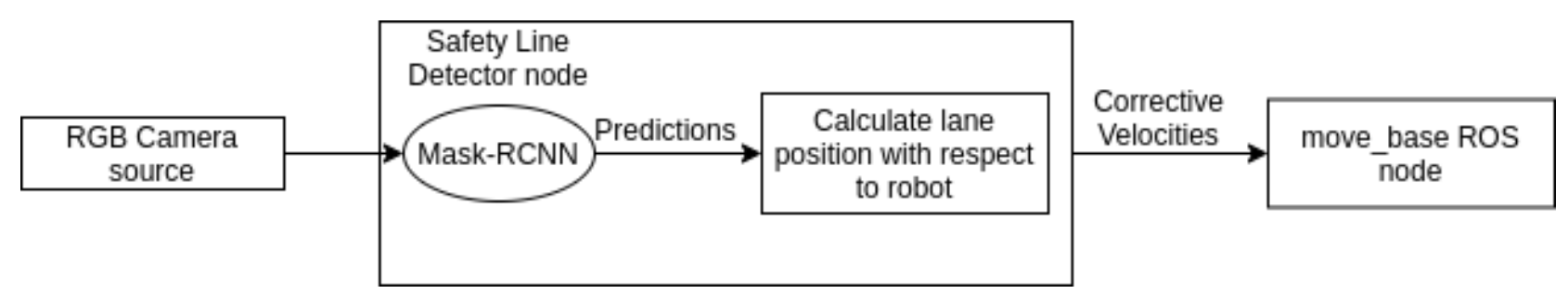

Fig. 2. ROS Node Architecture

As the robot uses the ROS operating system, a wrapper for the model must be introduced. A brief architecture of the node is displayed in Fig. 2. Firstly, we create a node that listens to robot's camera stream. That node also contains a member variable of our neural network model's class. The camera stream is also converted to a data-type acceptable by our model and then is fed to the model. The output of the model is a list of predictions containing the masks of the identified safety lines. Since, the robot should travel near the safety line, one can calculate how far the line is from the centre of the image. If it is furthest from a certain threshold, the velocities are corrected and sent to robot's drivers in order to retain the safety line as centred as possible in the current frame.

Another useful feature of the wrapper is its ability to turn the safety line detection on and off, in order to save computation resources as well as to expand the robot's battery life. This is applicable by using the ROS Framework and its services module.

\section{Experimental results}

The entire system is built on top of ROS [24] ecosystem. ROS is a middleware suite that provides various packages and software frameworks for easy robot software development. It also provides ready to use tools, that enable the robot to communicate, sense and act (indicatively tools for localization, mapping, visualization, debugging).

All simulations were conducted in a custom-made Gazebo simulation (Fig. 3) and the real-world experiments used the Turtlebot 2 with a kobuki base, equipped with RPLidar A3 sensor and Intel RealSense D435i camera sensor. The laptop that was used for controlling the robot has the following specifications: Intel Core i7-8750h CPU @ 2.2 GHZ, an Nvidia GeForce GTX 1060 and 8 GB RAM.

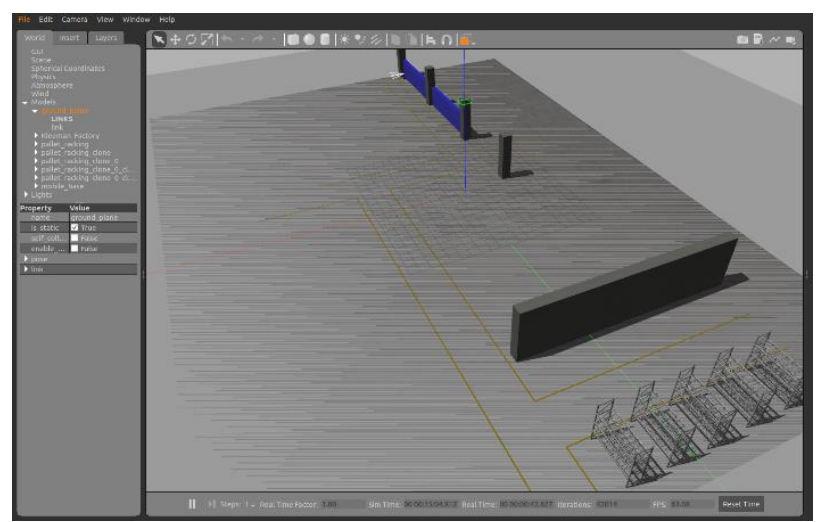

Left: Custom world environment

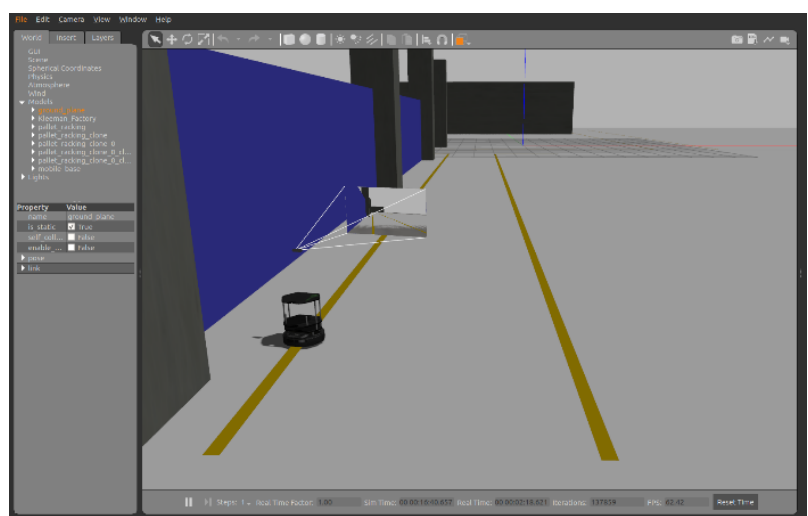

Right: Close up Turtlebot 2, equipped with camera

Fig. 3. Gazebo Simulation Environment

Gazebo environment simulates an industrial environment and is based on a real-world one. The only difference is that our simulation world has no dynamic or static objects like in the real-world one. Nevertheless, it is a good starting point to test if our node, safety line detection and course correction is working. To better visualize the results, the RViz tool is used. As we can see in Fig. 4, our model detects the mask of safety lines and for presenting the results we overlap them with the original image. We then procced to give a move goal to the robot, generating a path from its current pose to the goal. As the robot is moving and follows the path produced by the global planner, the corrected velocities are provided from the developed node and enable the vehicle to stay near the safety line. 

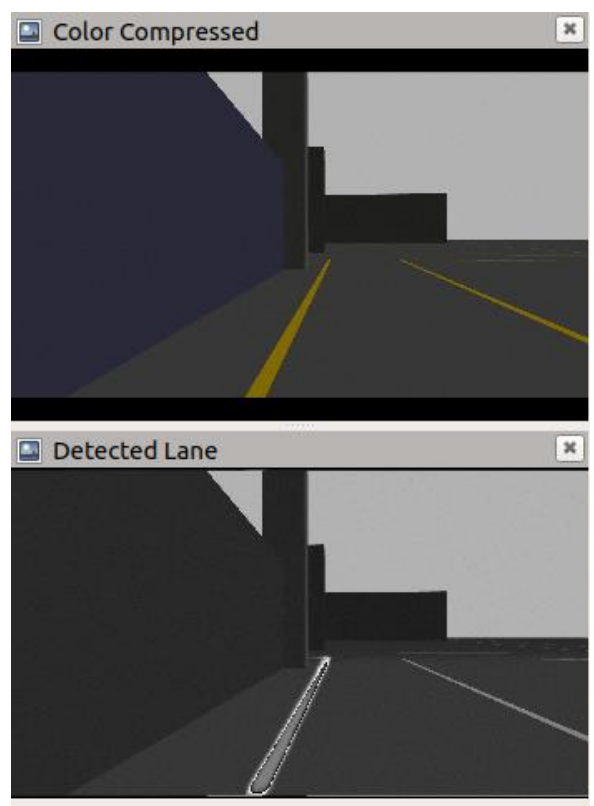
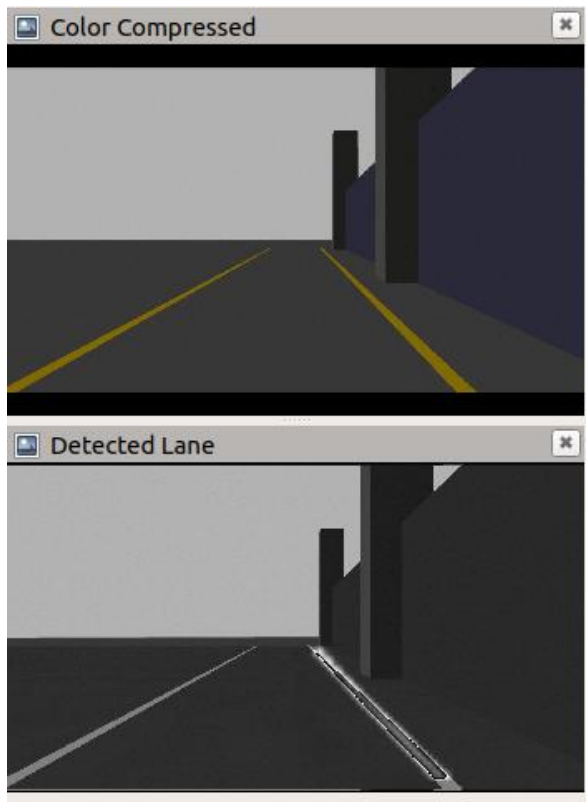

Fig. 4. Two snapshots of detected lines.

Likewise, when the node was used in the real-life scenario, the robot was able to detect the safety lines and keep close distance to them.
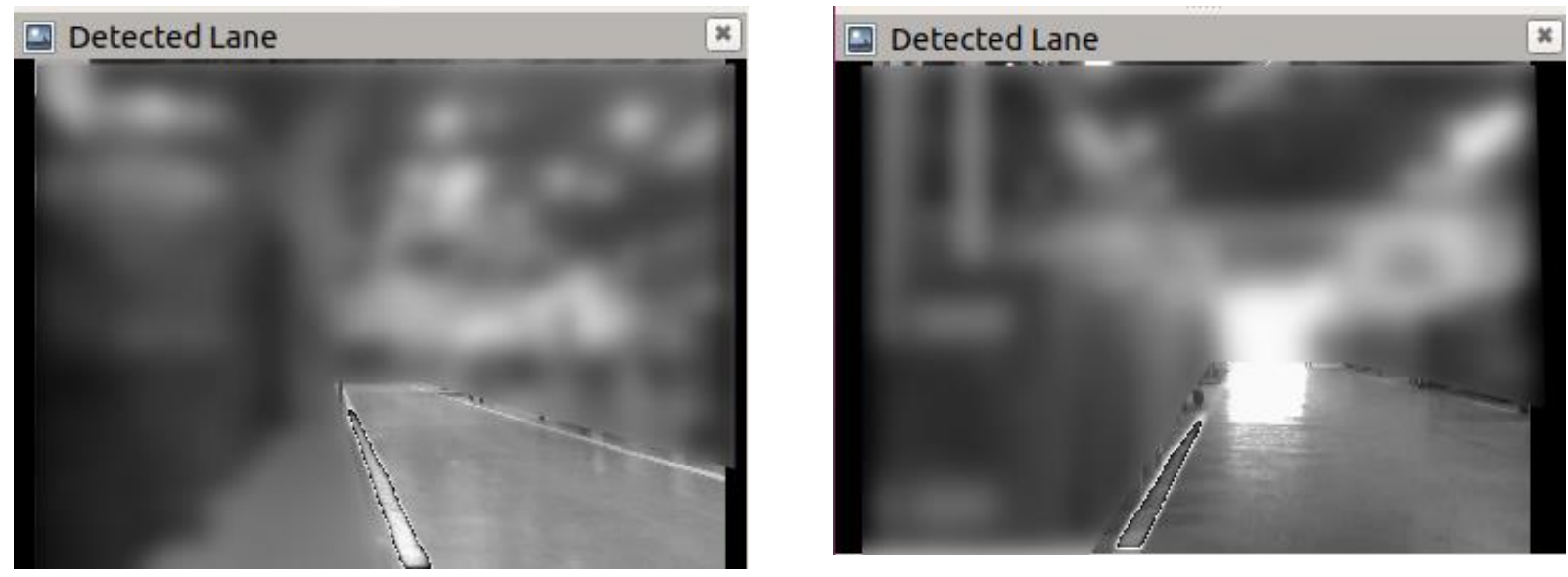

Fig. 5. Two snapshots of detected lines in real warehouse environment

The inference time of a single frame in the system described above is about 0.14 seconds making it almost suitable for real-time operation. Inferencing continuously though on the latest frame, resulted to some dropped frames and safety line following was unreliable. Instead, we operated our node at a fixed rate of $2 \mathrm{HZ}$ in order to improve reliability.

\section{Conclusion}

In this article, the authors proposed a method for safe navigation in industrial facilities. A lane detection system is introduced based on state-of-the-art Mask-RCNN model for AMR systems. Lane detection is a realistically identified as feasible method since floor markings are found in most industrial facilities and warehouses. The system is trained on manually annotated frames containing floor markings. Following the lane corrective commands are provided to the AMR to closely follow the line. The whole system is implemented on top of ROS and PyTorch frameworks. Experiments has shown that the system, can achieve almost-real time performance and that the AMR is successfully following the lanes' line.

Future work could incorporate the lanes detection system into an industrial SLAM (Simultaneous Localization and Mapping) solution. Mapping the floor markings, would greatly improve the localization and semantic information produced by SLAM system. Moreover, the system can be further improved, by training on more data from industrial facilities using multiple line types. 


\section{Acknowledgments}

This paper was partially supported by the Greek Secretariat of Research and Technology, Operational Programme Competitiveness, Entrepreneurship and Innovation 2014-2020, project "Dynamic Quality CONtrol on Production lines using intelligent AutonomouS vehicleS (Q-CONPASS)".

\section{References}

[1] Fragapane, G.; Ivanov, D.; Peron, M.; Sgarbossa, F.; Strandhagen, J. O. (2020). Increasing flexibility and productivity in Industry 4.0 production networks with autonomous mobile robots and smart intralogistics, Annals of Operations Research 2020, 1-19. doi:10.1007/S10479-020-03526-7

[2] Bechtsis, D.; Tsolakis, N.; Vlachos, D.; Srai, J. S. (2018). Intelligent Autonomous Vehicles in digital supply chains: A framework for integrating innovations towards sustainable value networks, Journal of Cleaner Production, Vol. 181, 60-71. doi:10.1016/J.JCLEPRO.2018.01.173

[3] Karamanos, X.; Mallioris, P.; Poulimenos, D.; Bechtsis, D.; Vlachos, D. (2019). A ros tool for optimal routing in intralogistics, Annals of DAAAM and Proceedings of the International DAAAM Symposium (Vol. 30). doi:10.2507/30th.daaam.proceedings. 115

[4] Hirano, Hiroyuki. (1995). 5 pillars of the visual workplace : the sourcebook for 5S implementation, 353

[5] Joshi, A. A. (n.d.). A Review on Seven S (7S) as a tool of Workplace Organization

[6] Gonzalez, J. P.; Ozguner, U. (2000). Lane detection using histogram-based segmentation and decision trees, IEEE Conference on Intelligent Transportation Systems, Proceedings, ITSC, 346-351. doi:10.1109/ITSC.2000.881084

[7] Hu, J.; Xiong, S.; Sun, Y.; Zha, J.; Fu, C. (2020). Research on Lane Detection Based on Global Search of Dynamic Region of Interest (DROI), Applied Sciences 2020, Vol. 10, Page 2543, Vol. 10, No. $7,2543$. doi:10.3390/APP10072543

[8] Ozgunalp, U.; Fan, R.; Ai, X.; Dahnoun, N. (2017). Multiple Lane Detection Algorithm Based on Novel Dense Vanishing Point Estimation, IEEE Transactions on Intelligent Transportation Systems, Vol. 18, No. 3, $621-632$. doi:10.1109/TITS.2016.2586187

[9] Niu, J.; Lu, J.; Xu, M.; Lv, P.; Zhao, X. (2016). Robust Lane Detection using Two-stage Feature Extraction with Curve Fitting, Pattern Recognition, Vol. 59, 225-233. doi:10.1016/J.PATCOG.2015.12.010

[10] Zhou, S.; Jiang, Y.; Xi, J.; Gong, J.; Xiong, G.; Chen, H. (2010). A novel lane detection based on geometrical model and Gabor filter, IEEE Intelligent Vehicles Symposium, Proceedings, 59-64. doi:10.1109/IVS.2010.5548087

[11] Jung, S.; Youn, J.; Sull, S. (2016). Efficient lane detection based on spatiotemporal images, IEEE Transactions on Intelligent Transportation Systems, Vol. 17, No. 1, 289-295. doi:10.1109/TITS.2015.2464253

[12] Wang, H.; Raj, B. (2017). On the Origin of Deep Learning

[13] Pizzati, F.; Allodi, M.; Barrera, A.; García, F. (2019). Lane Detection and Classification using Cascaded CNNs, Lecture Notes in Computer Science (Including Subseries Lecture Notes in Artificial Intelligence and Lecture Notes in Bioinformatics), Vol. 12014 LNCS, 95-103

[14] Neven, D.; de Brabandere, B.; Georgoulis, S.; Proesmans, M.; van Gool, L. (2018). Towards End-to-End Lane Detection: An Instance Segmentation Approach, IEEE Intelligent Vehicles Symposium, Proceedings, Vols. 2018June, 286-291. doi:10.1109/IVS.2018.8500547

[15] Paszke, A.; Chaurasia, A.; Kim, S.; Culurciello, E. (2016). ENet: A Deep Neural Network Architecture for RealTime Semantic Segmentation

[16] He, K.; Gkioxari, G.; Dollár, P.; Girshick, R. (2017). Mask R-CNN, IEEE Transactions on Pattern Analysis and Machine Intelligence, Vol. 42, No. 2, 386-397

[17] Dutta, A.; Zisserman, A. (n.d.). The VIA Annotation Software for Images, Audio and Video

[18] Lin, T.-Y.; Maire, M.; Belongie, S.; Bourdev, L.; Girshick, R.; Hays, J.; Perona, P.; Ramanan, D.; Zitnick, C. L.; Dolí, P. (n.d.). Microsoft COCO: Common Objects in Context

[19] Abadi, M.; Barham, P.; Chen, J.; Chen, Z.; Davis, A.; Dean, J.; Devin, M.; Ghemawat, S.; Irving, G.; Isard, M.; Kudlur, M.; Levenberg, J.; Monga, R.; Moore, S.; Murray, D. G.; Steiner, B.; Tucker, P.; Vasudevan, V.; Warden, P.; Wicke, M.; Yu, Y.; Zheng, X. (2016). TensorFlow: A system for large-scale machine learning, Proceedings of the 12th USENIX Symposium on Operating Systems Design and Implementation, OSDI 2016, 265-283

[20] Paszke, A.; Gross, S.; Massa, F.; Lerer, A.; Bradbury, J.; Chanan, G.; Killeen, T.; Lin, Z.; Gimelshein, N.; Antiga, L.; Desmaison, A.; Köpf, A.; Yang, E.; DeVito, Z.; Raison, M.; Tejani, A.; Chilamkurthy, S.; Steiner, B.; Fang, L.; Bai, J.; Chintala, S. (2019). PyTorch: An Imperative Style, High-Performance Deep Learning Library, Advances in Neural Information Processing Systems, Vol. 32

[21] Ren, S.; He, K.; Girshick, R.; Sun, J. (2015). Faster R-CNN: Towards Real-Time Object Detection with Region Proposal Networks, IEEE Transactions on Pattern Analysis and Machine Intelligence, Vol. 39, No. 6, 1137-1149

[22] He, K.; Zhang, X.; Ren, S.; Sun, J. (2015). Deep Residual Learning for Image Recognition, Proceedings of the IEEE Computer Society Conference on Computer Vision and Pattern Recognition, Vols. 2016-December, 770-778

[23] Redmon, J.; Farhadi, A. (n.d.). YOLOv3: An Incremental Improvement

[24] Quigley, M.; Gerkey, B.; Conley, K.; Faust, J.; Foote, T.; Leibs, J.; Berger, E.; Wheeler, R.; Ng, A. (n.d.). ROS: an open-source Robot Operating System 